Parkinson's disease

\section{Driving in Parkinson's disease}

\section{A Schrag}

It is not possible for patients to predict their driving safety from a motor examination or the patient's own judgement

$P$ atients with Parkinson's disease are usually advised to inform the driving licensing authority and their driving insurance company of the diagnosis. However, until a few years ago, Parkinson's disease was not considered a major obstacle to safe driving until the advanced stages. This changed when in 1999 sudden onset sleep attacks in patients with Parkinson's disease on dopaminergic drugs were first reported. This not only led to considerable concern about the safe use of dopaminergic drugs in patients who drive, but also to a flurry of studies investigating the frequency of the problem, the contributing factors, and the mechanism and types of drugs associated with it. However, while this remains an important issue, relatively little attention has been paid to the overall ability and competence of patients with Parkinson's disease to drive a motor vehicle, unrelated to sleep attacks.

In this edition of JNNP (see $p p$ 17680 ), Wood et $\mathrm{al}^{2}$ address this issue using structured qualitative and quantitative assessments of driving ability in patients with Parkinson's disease and age matched controls. Experienced, qualified driving assessors who were blinded to the diagnosis found that in a realistic, on road setting, overall driving safety in patients with Parkinson's disease was significantly impaired; worryingly, a high proportion (more than 50\%) of patients would not have passed a state based driving test. The study included 25 patients who had volunteered for the study after attending lectures on the subject, and it is therefore not possible to conclude that such a high percentage of the overall population of patients with Parkinson's disease would fail a driving test. However, it highlights the potential impairment of driving competence that patients with Parkinson's disease may experience.

An important finding was that the driving examiner's assessment of driving ability correlated poorly with the drivers' own view of how well they were driving. Poor driving performance also did not correlate with disease severity as measured on clinical scales, although longer disease duration was associated with greater difficulties. No neuropsychological tests were included in the study, but the type of errors made related not only to difficulties in motor tasks but also appeared to be related to the neurocognitive deficits known to occur in Parkinson's disease. ${ }^{3}$ Thus, while slowness of movement and reaction time are likely to play important roles in driving performance, other factors such as impaired visuo-spatial processing, planning, and sequencing are also likely to contribute to the difficulties.

While these results must be confirmed in larger, unselected samples of patients with Parkinson's disease who are currently driving, the present study not only draws attention to the impairment of driving ability in Parkinson's disease but also shows that it is not possible for patients to predict their driving safety or to deduce their driving ability from motor examination. While the recommendation to stop driving (or to have a driving assessment) is currently often based on physicians' overall clinical judgment, this study highlights the need to assess driving ability in Parkinson's disease regularly and in a standardised fashion. It also calls for studies to explore the motor and nonmotor factors leading to impaired driving performance and whether compensatory strategies may help patients improve their driving ability and maintain independence for longer.

J Neurol Neurosurg Psychiatry 2005;76:159. doi: 10.1136/jnnp.2004.053694

Correspondence to: Dr Anette Schrag, Department of Clinical Neurosciences, Royal Free Hospital, University College London, London NW3 2QG, UK; a.schrag@medsch.ucl. ac.uk

Competing interests: None declared

\section{REFERENCES}

1 Frucht S, Rogers JD, Greene PE, et al. Falling asleep at the wheel: motor vehicle mishaps in persons taking praimpexole and ropinirole. Neurology 1999;52:1908-10.

2 Wood JM, Worringham C, Kerr G, et al. Quantitative assessment of driving performance in Parkinson's disease. J Neurol Neurosurg Psychiatry 2004;75:176-80.

3 Emre M. What causes mental dysfunction in Parkinson's disease? Move Disord 2003;18(suppl 6):S63-71.

sive decrease of the fractional anisotropy (FA) with an increase in mean diffusivity $(\mathrm{MD})$ in the pyramidal tract at the level of the cerebral peduncle, reflecting the changes expected in WD-a progressive disintegration of fibre structure. Hervé et $a l^{2}$ serially studied nine patients from 1 week to 6 months following MCA territory stroke, focusing on the ipsilateral thalamus. They found significant increases in MD from 1 month onward, without parallel changes in FA, presumably reflecting a progressive loss of neurones and/or glial cells. These changes in thalamic MD without changes in FA indicate that within complex multi-nucleated grey matter structures devoid of large fibre bundles such as the thalamus, changes in FA should not be anticipated while loss of organised cellular arrangements will 
translate as increased MD. These findings likely reflect retrograde degeneration of the thalamo-cortical neurones secondary to fibre damage, known to translate in its early stage as hypometabolism and microglial activation in positron emission tomography (PET) studies using the tracers ${ }^{18} \mathrm{FDG}$ and ${ }^{11} \mathrm{C}-\mathrm{PK} 11195$, respectively, ${ }^{34}$ and in its late stage as reduced thalamic volume on structural imaging (also observed by Hervé et al).

Fractional anisotropy-the degree to which diffusion is anisotropic-reflects the parallel arrangement of fibres, so is used as an index of integrity for any particular bundle. However, this method is limited because to obtain quantitative values for a given fibre tract, a region of interest must be positioned within the bundle based on a priori knowledge. A sophisticated application of DTI referred to as tractography applies mathematical modelling to three dimensional anisotropy data to map the trajectory of a fibre in both directions from a chosen "seed" point in the bundle. Among the various tractography techniques, one particularly promising approach takes into account all possible directions rather than just the main eigenvector, which in turn allows the probabilistic mapping of connections between gray matter areas, including cortical to deep nuclei and vice versa. ${ }^{5}$ Exquisite mapping of the thalamo-cortical connection systems has been obtained using this method; the application to stroke is eagerly awaited.

But what purposes will the mapping of fibre and cell degeneration after stroke serve? Whilst studies such as these are just the beginnings, several potential uses of this new approach are emerging. Quantifying with FA the degree of damage to a single well identified tract such as the pyramidal may, in conjunction with functional imaging such as fMRI, help to understand the pathophysiological mechanisms underlying recovery. For instance, knowing this quantitative index of damage could allow one to derive in quantitative terms the amount of recovery that can be ascribed to adaptive plasticity. ${ }^{6}$ Also, estimating the number of fibres connecting two or more cortical centres may help to determine the occurrence of functional versus anatomical disconnection. However, because the effects measured with DTI only reflect secondary effects of stroke that develop as patients recover, no obvious direct clinical implication of these studies has emerged thus far, and accordingly Hervé et $a l^{2}$ found no significant correlation between the changes they measured in the ipsilateral thalamus and concomitant clinical scores or changes thereof. Although these remote degeneration effects might therefore be epiphenomenal, one may envisage that they may impede or slow down recovery if they were to impact on intact but inter-connected structures for example. Whilst arguably a long shot, one could in turn wonder whether arresting or even preventing secondary degeneration could be worthwhile - although by doing so one might perhaps worsen rather than enhance outcome if the

\section{Thrombolysis for central retinal artery occlusion}

\section{G T Plant, K Landau}

\section{Results of a controlled trial are needed before recommending this treatment}

( entral retinal artery occlusion is an uncommon cause of unilateral visual loss and a rare cause of blindness. In this issue (see $p p$ 196-9), Arnold et al ${ }^{1}$ describe a retrospective analysis of intra-arterial thrombolysis (IAT) of central retinal artery occlusion within six hours of symptom onset in 37 patients. The visual outcome was compared with 19 control patients. Before treatment, cranial computed tomogra- degeneration served to re-set the local excitatory/inhibitory balance. ${ }^{7}$ More work is obviously needed to address these issues but we now at last have a tool at our disposal to quantify secondary degeneration after stroke and to monitor any effect of intervention in parallel with clinical function.

\section{J Neurol Neurosurg Psychiatry}

2005;76:159-160.

doi: 10.1136/jnnp.2004.051870

Correspondence to: Professor JeanClaude Baron, University of Cambridge, Department of Neurology, Addenbrookes Hospital, Box 83, Cambridge CB2 2QQ, UK; jcb54@cam.ac.uk

Competing interests: none declared

\section{REFERENCES}

1 Thomalla G, Glauche V, Weiller C, et al. Time Course of Wallerian Degeneration after Ischemic Stroke Revealed by Diffusion Tensor Imaging. J Neurol Neurosurg Psychiatry 2004;75:266-8.

2 Hervé D, Molko N, Pappata S, et al. Longitudinal thalamic diffusion changes after middle cerebral artery infarcts. J Neurol Neurosurg Psychiatry 2004;75:200-5

3 Baron JC. Depression of energy metabolism in distant brain structures: studies with positron emission tomography in stroke patients. Semin Neurol 1989;9:281-5.

4 Pappata S, Levasseur M, Gunn RN, et al. Thalamic microglial activation in ischemic stroke detected in vivo by PET and 11C-PK1 1,195. Neurology 2000;55:1052-4.

5 Behrens TE, Johansen-Berg H, Woolrich MW, et al. Non-invasive mapping of connections between human thalamus and cortex using diffusion imaging. Nat Neurosci 2003;6:750-7

6 Calautti C, Baron JC. Functional imaging of motor recovery after stroke: a review. Stroke 2003;34:1553-66.

7 Schallert T, Jones TA, Lindner MD. Multilevel transneuronal degeneration after brain damage: behavioral events and effects of anticonvulsant gamma-aminobutyric acid-related drugs. Stroke 1990;21(Suppl III):143-6.

hours could not be treated because no neuroradiologist was available. Periprocedural adverse events occurred in two patients who experienced transient ischaemic attacks and in one patient who suffered brain infarction. No haemorrhagic complications were seen. The outcome measure was visual acuity.

Thrombolysis in central retinal artery occlusion is not new ${ }^{3}$ but it is Deiter Schmidt and Martin Schumacher in Freiburg, Germany, who have championed the technique, ${ }^{4}$ treating over 60 cases $^{5}$ and compelling us to examine our practice. IAT to treat ischaemic stroke is a goal of stroke units; however, the time interval between event and intervention is critical. Sudden visual loss tends to present to eye casualty departments. In cases of acute blindness a competent ophthalmic examination is essential but will increase the "time to treat", especially where the eye hospital is a standalone unit.

Who is doing it? In the United Kingdom a few anecdotal cases are 
described at meetings but only over coffee. In the USA the procedure is being done, but no systematic information is available (Miller $\mathrm{N}$ and Aldrich E, personal communication). In contrast IAT for central retinal artery occlusion is commonplace in Germany, Switzerland, and Austria. In Zurich, for example, 88 consecutive patients with central retinal artery occlusion have been treated between 1995 and 2002: IAT was undertaken in 23 patients while 65 were managed conservatively (unpublished data). A trend for a better visual acuity outcome was observed in the thrombolysis group, which became statistically significant for younger patients who had slightly better initial visual acuities. A small subgroup of patients profited significantly from IAT, all of whom were treated within six hours. Unknown factors other than the time to thrombolysis contribute to the outcome.

It is not our opinion that the results presented by Arnold et al should encourage centres to begin using this treatment in advance of a randomised study. Such a study is under way in Europe (no UK centre is involved but the acronym is English: EAGLE, European assessment group for lysis in the eye).

Our reasons for this view are as follows. First, central retinal artery occlusion is usually a monocular disorder and therefore (apart from the risk of pre-existing or subsequent pathology in the fellow eye) the likelihood of significant disability is less than with cerebral arterial occlusions.

Second, the logistic problems associated with setting up a protocol for IAT in central retinal artery occlusion are substantial. In our experience patients often do not notice monocular visual loss for some time or may "wait for it to clear" before seeking medical advice. Furthermore the additional review by an ophthalmologist mentioned above adds time compared with the journey of a patient with a hemisphere stroke. Furthermore, many patients wake with loss of vision, so how can we know when the event occurred?

Third, there is no information in this study on the extent of visual field loss as an outcome measure. This is significant for disability and may not correlate well with visual acuity because acuity may be preserved where a cilioretinal artery is present and with residual retinal branch artery based defects.
Fourth, there was a low level of statistical significance in the study under review.

Finally, Arnold et al defined a subclass of patients with central retinal artery occlusion who would have a greater chance of benefiting from IAT. In the light of a trend in their own data, existing animal studies, and recommendations for thrombolysis in cerebral ischaemic stroke, they suggest that treatment within three hours might be the goal.

The following analysis should be carried out in a large city:

- The incidence of unilateral central retinal artery occlusion needs to be established.

- A logistic study should be undertaken of the likelihood of such patients presenting to an interventional neuroradiological unit within three hours having undergone an ophthalmological examination, a medical/neurological examination, brain neuroimaging to exclude haemorrhage (but is this necessary in central retinal artery occlusion as opposed to hemisphere strokes?), sufficient blood tests with laboratory results available to exclude vasculitis and blood dyscrasias, and four-vessel interventional angiography to ensure adequate access to the ophthalmic artery.

- The additional cost to eye departments and stroke units of maintaining a 24 hour referral and treatment service for central retinal artery occlusion should be estimated, as should the geographical density required for such units, taking into account the results of the first two investigations above.

- A meta-analysis of existing studies should be done, including those employing intravenous treatment and other thrombolytic agents.

- The number of cases required should be determined, given the statistical significance in the existing retrospective studies, to permit not only a definitive overall result but also stratification of the cases according to time to thrombolysis, age, and other clinical features such as the type of embolus where visible.

- Perimetry should be evaluated as an outcome measure.

- Evaluation of visible emboli is required: can thrombolysis be effective in a patient with a calcific embolus sitting at the bifurcation of the central retinal artery as opposed to a local thrombosis?

- An evaluation should be made of the presence or absence of a macular cilio-retinal artery, which will influence preservation of central acuity but not necessarily disability (patients with a macular cilio-retinal artery are often left with $6 / 9$ acuity but a tiny central island of vision of little practical use).

As neuro-ophthalmologists our aim is to preserve vision wherever possible. There are times when the task of obtaining the evidence we need seems impossible. However, we should remember the words of Louis XVI's finance minister, Charles Alexandre de Calonne: "Madame, si c'est possible, c'est fait; impossible? Cela se fera"-better known as the US Army paraphrase "The difficult we do immediately; the impossible takes a little longer." As Calonne discovered, it sometimes takes a little too long.

J Neurol Neurosurg Psychiatry 2005;76:160-161.

doi: 10.1136/jnnp.2004.045583

\section{Authors' affiliations}

G T Plant, The National Hospital for Neurology and Neurosurgery, Queen Square, London WCl, UK

K Landau, Department of Ophthalmology, University Hospital Zurich, Frauenklinikstrasse 24, Zurich, Switzerland

Correspondence to: Gordon T Plant, Department of Neurology, The National Hospital for Neurology and Neurosurgery, Queen Square, London WCIN 3BG, UK; gordon@plant.globalnet.co.uk

Competing interests: none declared

\section{REFERENCES}

1 Arnold M, Körner U, Remonda L, et al. Comparison of intra-arterial thrombolysis with conventional treatment in patients with acute central retinal artery occlusion. J Neurol Neurosurg Psychiatry 2004;75: 196-9.

2 Adams HP, Davis PH, Leira EC, et al. Baseline $\mathrm{NIH}$ stroke scale score strongly predicts outcome after stroke: a report of the trial of Org 10172 in acute stroke treatment (TOAST). Neurology 1999;53:126-31.

3 Ffytche TJ. A rationalization of treatment of central retinal artery occlusion. Trans Ophthalmol Soc UK 1974;94:468-79.

4 Schumacher M, Schmidt D, Wakhloo AK. Intraarterielle Fibrinolyse bei Zentralarterienverschluss. Radiologe 1991;31:240-3.

5 Schmidt DP, Schulte-Mönting J, Schumacher M. Prognosis of central retinal artery occlusion: local intra-arterial fibrinolysis versus conservative treatment. Am J Neuroradiol 2002;23:1301-7. 